\title{
Comparison of conduit and autograft efficiency in repairing femoral nerve injury in New Zealand rabbits
}

\author{
Seyed Mehdi Moosavizadeh ${ }^{1}$, Hamidreza Alizadeh Otaghvar*2, Mohammad Baghae ${ }^{3}$, \\ Arefeh Zavari ${ }^{4}$, Hamid Mohyeddin ${ }^{5}$, Hamidreza Fattahiyan ${ }^{6}$, Behnood Farazmand ${ }^{4}$, \\ Seyed Mohammad Ali Moosavizadeh ${ }^{7}$
}

\section{Abstract}

Background: Peripheral nerve injuries may affect all age groups and exert devastating impacts on the professional and personal life of the patients. The investigation of nerve regeneration and use of biomaterials and synthetic materials have resulted in advancements in the treatment of peripheral nerve injuries and lesions. Nerve conduits can be used to adjoin the digital sensory nerve spaces of less than $3 \mathrm{~cm}$, especially when the direct tension-free repair of peripheral nerve lesions is not possible. The present study was conducted to evaluate the use of nerve conduits by functional and nonfunctional parameters (i.e. histological study).

Methods: This experimental study was conducted on 30 male rabbits. After cutting or crushing the right femoral nerve of the rabbits, they were divided into 3 groups: group 1, with right femoral nerve cut; group 2, with right femoral nerve crushed; and group 3, with right femoral nerve cut using a conduit. The 3 groups were evaluated after 1,8 , and 16 weeks for functional parameters (i.e. walking track analysis). In addition, they were subjected to nonfunctional examination (i.e. histological study) after 16 weeks, then, the results were compared.

Results: The 3 groups showed no statistically significant differences in motor recovery in the eighth and 16th weeks ( $p>0.05$ ). Based on the histological study, group 3 with an end-to-end nerve cutting using a conduit, showed a significantly higher axon count compared to groups 2 and $3(\mathrm{p}<0.05)$.

Conclusion: End-to-end anastomosis using conduit led to axon growth; moreover, comparable functional recovery was observed with end-to-end neurorrhaphy in a rabbit model. Given that the diameter of the nerves and muscles, which might be neurotized in humans, and is much bigger and not comparable to that of the rabbits, it is highly recommended to conduct studies on animals with the larger size, such as primates, to facilitate the generalization of the results to humans.

Keywords: Conduit, Femoral nerve, Nerve anastomosis, Motor recovery, Histologic study

Conflicts of Interest: None declared

Funding: A grant obtained from Iran University of Medical Sciences

*This work has been published under CC BY-NC-SA 1.0 license.

Copyright $\subseteq$ Iran University of Medical Sciences

Cite this article as: Moosavizadeh SM, Alizadeh Otaghvar H, Zavari A, Mohyeddin H, Fattahiyan H, Moosavizadeh SMA, Farazmand B, Baghae M. Comparison of conduit and autograft efficiency in repairing femoral nerve injury in New Zealand rabbits. Med J Islam Repub Iran. 2018 (13 Oct);32:99. https://doi.org/10.14196/mjiri.32.99

\section{Introduction}

Peripheral nerve injuries affect all age groups and can have adverse impacts on the patients' professional and personal life as well as activities. Peripheral nerve injuries repeatedly occur as a result of trauma, surgery, or acute compression. These injuries can be caused by traumatic

Corresponding author: Dr Hamidreza Alizadeh Otaghvar, drhralizade@yahoo.com

1. Department of Plastic Surgery, 15 Khordad Educational Hospital, School of Medicine, Shahid Beheshti University of Medical Sciences, Tehran, Iran

2. Trauma and Injury Research Center, Iran University of Medical Sciences, Tehran, Iran

3. Shahid Beheshti University of Medical Sciences, Trauma and Injury Research Center, Tehran, Iran

4. Iran University of Medical Sciences, Tehran, Iran.

5. Department of Clinical Sciences, Faculty of Veterinary Medicine, Garmsar Branch, Islamic Azad University, Garmsar, Iran

6. Department of Clinical Sciences, Faculty of Specialized Veterinary Science, Science and Research Branch, Islamic Azad University, Tehran, Iran

7. Shahid Beheshti University of Medical Sciences, Tehran, Iran events, such as a traumatic open fracture and wound. In addition, they can present latently after a peripheral nerve block or during a compartment syndrome.

Generally, peripheral nerve injuries should be managed immediately in clinics. The cut and repaired nerve ends

\section{$\uparrow$ What is "already known” in this topic:}

Conduit may help nerve sprouting in a right direction. However, many studies have investigated sensory and mixed nerves, which mostly compared anastomosis and conduit.

$\rightarrow$ What this article adds:

This study proposed that even in situations where end-to-end anastomosis and nerve repair (e.g. crushed nerves or newly found cut ended nerves) is not possible, it is preferable to use conduit. 
are subjected to different mechanical forces that must be recognized and conducted appropriately to preserve blood flow and allow nerve healing (1). In the peripheral nervous system, Schwann cells make a great contribution to axonal regeneration after nerve injury. According to the evidence, in mammals, the Schwann cells decrease the expression of myelin following the nerve injury, and the myelin sheath is lost. On the other hand, an increase occurs in the expression of transcription factors accompanied by an immature Schwann cell state $(1,2)$.

Furthermore, on the first-day post-injury, the Schwann cells spontaneously process and eliminate myelin debris in intracellular vacuoles and expose the extracellular myelin debris for engulfment by macrophages. There are multiple treatment options to manage peripheral nerve injuries. These therapeutic options include immediate or late repair, nerve grafting, nerve transfer, nerve transplant, nerve conduits, and cell therapy. Moreover, the inoculation of cells into the injury sites can accelerate and progress the quality of nerve regeneration.

Advances in the treatment of peripheral nerve lesions are the result of the investigation of nerve regeneration and use of biomaterials and synthetic materials. When the direct tension-free repair of peripheral nerve lesions is not possible, nerve conduits can be used to bridge the digital sensory nerve gaps of $\leq 3 \mathrm{~cm}$. Nerve autograft is a benchmark for larger, longer, mixed, or motor nerve defects.

Autogenous biological conduits (i.e. commonly veins and, rarely arteries) are applicable in the nerve gaps of $<3$ $\mathrm{cm}$ in length. The conceptualization of cellular changes subsequent to nerve injury is a matter of significant importance in determining proper timing and technique for nerve repair to achieve optimal functional outcomes $(3,5,6)$

On the other hand, femoral neuropathies, playing a key role in the motor function of the lower extremity, impose great disease burdens and medical expenses. Femoral nerve can be divided into 2 branches, one of which supplies the skin on the anteromedial thigh, and the other supplies the skin on the medial side of the leg and foot.

The investigation of the systemic and topographical anatomy of rabbits and other similar mammals have revealed that femoral nerve generally originates from the ventral branches of the fourth, fifth, sixth, and seventh lumbar spinal nerves both in males and females $(3,4)$.

Incorrect nerve anastomosis reduces the diameter of the nerve fiber and thickness of myelin sheaths in the regenerated nerve fibers. Direct nerve suture cannot effectively improve the functional recovery of the damaged nerves. On the other hand, nerve chemotaxis, coupled with nerve regeneration chamber, can efficiently improve the effects of nerve regeneration and enhance the prognosis of nerve injury repair $(8,10)$.

The use of autologous grafts makes it difficult to eliminate other methods and grafts to a large extent. Therefore, this study was conducted to compare the partial repair of the femoral nerve in rabbits with the other method, autologous femoral nerve graft to femoral vein conductor. The findings of this study could be of help in increasing the hope of these patients in the future.

\section{Methods}

This experimental study was conducted on 30 white male New Zealand rabbits purchased from the animal laboratory of Shahid Beheshti University, Tehran, Iran. The rabbits were 24-26 weeks old and had the mean height of $40.89 \mathrm{~cm}$ and weight range of 2-3.8 $\mathrm{kg}$. They were prepared for standard aseptic surgery and anesthetized through the intravenous administration of ketamine and xylazine (90 ng/kg ketazol $+10 \mathrm{mg} / \mathrm{kg}$ xylazine or promprone $+0.05 \mathrm{ng} / \mathrm{kg}$ atropine). The rabbits were randomly divided into 3 groups as follow:

In group 1 (right femoral nerve cut), the right femoral nerve was cut and the left femoral nerve remained intact ( $\mathrm{n}=10$ ); in group 2 (right femoral nerve crushed), the right femoral nerve was crushed, but not completely cut, and the left femoral nerve remained intact $(\mathrm{n}=10)$; and in group 3 (right femoral nerve cut using conduit), the right femoral nerve was cut, but a conduit was used for the regeneration process, and the left femoral nerve was intact $(n=10)$.

\section{Motor recovery-walking track analysis}

To elevate the femoral nerve recovery, walking track analysis was performed 1,8 , and 16 weeks post-surgery. To determine the walking pattern of the rabbits, their paws were pressed onto an inkpad. Subsequently, they were allowed to walk up a small inclined walkway (slope of 20length of $3 \mathrm{~m}$, width of $56 \mathrm{~cm}$ ), which was lined with white paper. All rabbits had few training runs.

The footprint parameters were measured as follow:

(1) Print length (the distance of the heel to the third toe);

(2) Toe spread (the distance of the first to the fifth toe);

(3) Intermediate toe spread (the distance of the second to the fourth toe).

All these measurements were performed considering the left experimental paw of each rabbit under study.

\section{Histological study}

In this study, 16 weeks post-surgery, tissue specimens were excised from the femoral nerve of the ischiocavernosus below the anastomosis sites in all experimental groups. The specimens were then fixed with paraformaldehyde $4 \%$ and stained with tetraoxide osmyiom 1\%. Subsequently, the nerves were stained with toluidine blue $1 \%$ and observed under a high-power microscope (Olympus AX70, Japan).

\section{Statistical procedure}

The description of the data was expressed as the mean and standard deviation. The statistical differences between the groups were analyzed using one-way ANOVA and repeated measure ANOVA used to compare the differences over the time (1st, 8th, 16th weeks postsurgery) within each group. The Kolmogorov Smirnov test was used to check the normality of distribution. Moreover, the Bonferroni post-hoc correction was used for multiple group comparison. The P-value of less than 0.05 was considered statistically significant. 


\section{Results}

Motor recovery-walking track analysis is a common method to assess motor nerve recovery after femoral nerve injury in rabbits by recording their footprints. Based on the statistical analysis of footprinting data, including print length, toe spread, and intermediate toe spread, no significant difference was observed in group 1 in the first, eighth, and $16^{\text {th }}$ weeks post-surgery.

On the other hand, the motor recovery of group 2 was significantly different in the first week compared to those

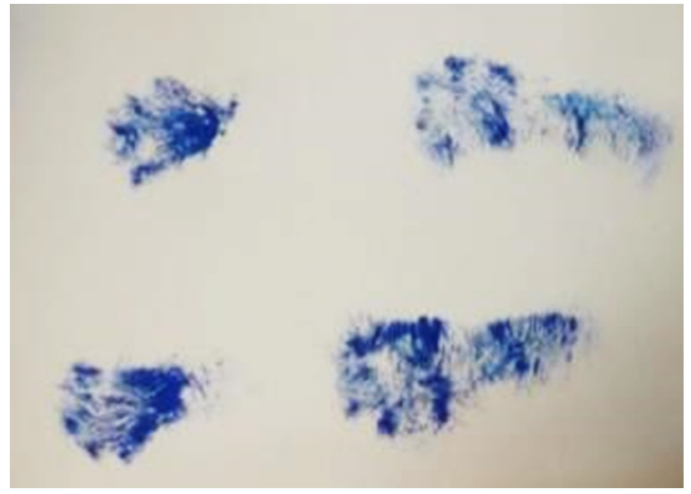

Fig. 1. A rabbit's footprints before the implementation of the experimental methods.

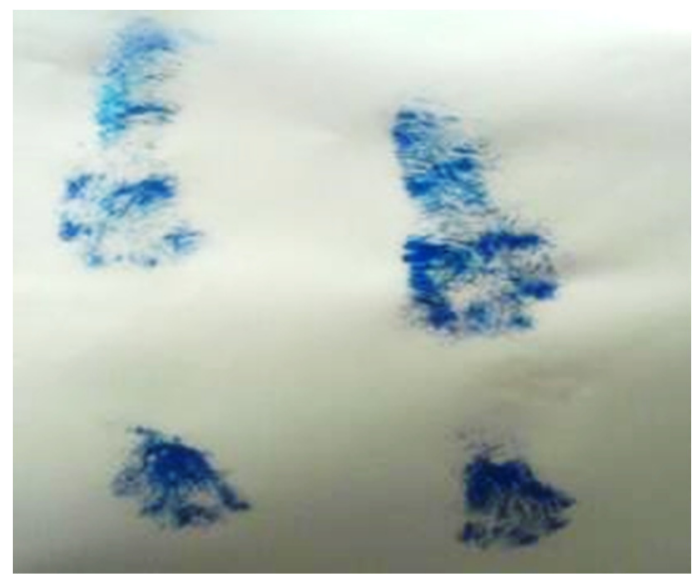

Fig.2. A rabbit's footprints 8 weeks post-surgery

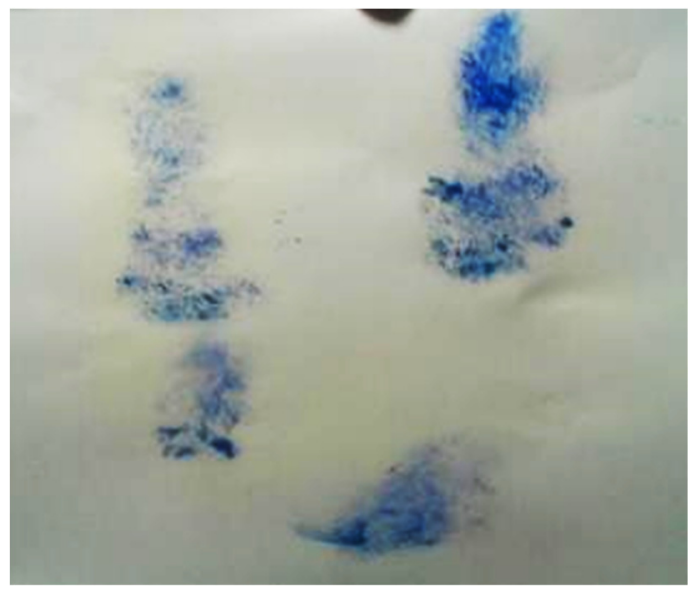

Fig. 3. A rabbit's footprints sixteen weeks post-surgery.
Table 1. Comparison of the 3 groups for mean print length, toe spread, and intermediate toe in the $16^{\text {th }}$-week post-surgery

\begin{tabular}{lccc}
\hline Footprint data & Group 1 & Group 2 & Group 3 \\
\hline $\begin{array}{l}\text { Mean print length } \\
(\mathrm{cm})\end{array}$ & $5.2(+/-1.5)$ & $4.8(+/-1)$ & $5.1(+1 /-1.5)$ \\
$\begin{array}{l}\text { p-value }=0.05 \\
\text { Mean toe spread } \\
(\mathrm{cm})\end{array}$ & $1.7(+/-0.5)$ & $1.08(+/-0.3)$ & $1.56(+/-0.4)$ \\
p-value $=0.05$ & & & \\
$\begin{array}{l}\text { Mean intermediate } \\
\text { toe spread }(\mathrm{cm})\end{array}$ & $1.38(+/-0.3)$ & $1.01(+/-0.2)$ & $1.034(+/-0.3)$ \\
p-value $=0.05$ & & & \\
\hline
\end{tabular}

in the eighth and $16^{\text {th }}$ weeks. However, this group showed no significant difference in this regard in the eighth week compared to the $16^{\text {th }}$ week post-surgery. Meanwhile, group 3 showed a statistically significant difference in the eighth and $16^{\text {th }}$ weeks post-surgery compared to the first week. Additionally, motor recovery was significantly different between the eighth and $16^{\text {th }}$ weeks in group 3 . There was a significant difference between group 1 and the other 2 groups in this respect. Furthermore, a statistically significant difference was observed between groups 2 and 3 eight weeks after surgery $(p=0.01)$.

Table 1 presents the comparison of the mean print length, toe spread, and intermediate toe 16 weeks postsurgery among the 3 groups. According to the results, there was no significant difference between groups 1 and 3 with respect to these variables.

Table 1 presents the comparison of the mean print length, toe spread, and intermediate toe 16 weeks postsurgery among the 3 groups. According to the results, there was no significant difference between groups 1 and 3 with respect to these variables.

\section{Histological results}

The examination of the cross sections performed 16 weeks after the surgery revealed that group 3, with an endto-end nerve cutting using a conduit, showed a significantly higher axon count compared to groups 2 and 3.

\section{Discussion}

The use of conduit for nerve repairing has been established as a treatment of choice. In peripheral nerve injuries with unavailable proximal stumps or long nerve defects, nerve repair can be potentially performed with autologous nerve graft to vein conductor technique. This technique for nerve repairing has recently attracted special attention in experimental practice. In a study, Jinniang Nan retrieved 793 publications on the use of nerve conduits for peripheral nerve injury repair from the Web of Science during 2002 and 2011. The number of publications gradually increased over a 10 -year period.

In a study investigating 28 rats, Yuki Iijima et al. (2016) reported that the vascularization of artificial nerve conduits accelerated the peripheral nerve regeneration. For the purpose of the study, they removed a 13-mm segment of the sciatic nerve and then pressed a heated iron against the dorsal thigh muscle to produce a burn. The defects were immediately repaired with an autograft $(n=10)$, 
nerve conduit graft $(n=8)$, or vascularized nerve conduit graft $(n=8)$. The recovery of motor function was examined in 18 weeks post-surgery (11).

Clavijo-Alvarez et al. examined 60 rats in 6 groups of control (contralateral leg samples; $n=10$ ), negative (nerve gap defect; $n=6)$, autograft $(n=10)$, polycaprolactone $(n=10)$, Cultiguides $(n=10)$, and neurotube $(n=10)$. They demonstrated a significant difference among the study groups in terms of the differences in myelination. In addition, the neurotube group had the highest degree of myelination $(\mathrm{P}<0.001)$ compared to other groups $(12,13)$.

Traditionally, the results of nerve repair have been considered to depend on the patient, injury, and technical factors. Injury factors include the age of the patient, the mechanism of the injury, and affected nerve (i.e. pure motor versus mixed) $(7,8)$. According to Saeid Kamal Frutan, the stimulation of neurotrophic factors arising from the recipient distal nerve stump may induce nerve sprouting (10).

To study the functional recovery of injured nerve, we compared the partial repair of the nerve with the other method, autologous nerve graft, to vein conductor in femoral nerve. Controversial findings have been reported in various studies on nerve conduction, such findings are mainly due to 2 factors. First, axonal regeneration is not selective; in other words, sometimes, despite good nerve regeneration, only sensory fibers regenerate into the recipient's nerve, and vice versa. Second, several factors, such as age, play a pivotal role in nerve repair.

Based on the findings of the present study, from the histological point of view, the autologous nerve graft method resulted in more favorable outcomes compared to the vein conductor. The neurotrophic factor is also the most important factor affecting the regeneration of injured axon. It seems that this factor had a high impact on using conduits for the repair of the nerves in group 3 compared to other groups. The difference in axon count in the partial repair of the nerves with the other method, autologous nerve graft, to the vein conductor seems to be increasing (9).

Based on our findings, the use of conduits to repair nerves led to axon growth. Moreover, a comparable functional recovery was observed with a partial repair in a rabbit model. The diameter of the nerves and muscles, which might be neurotized in humans, is much bigger and not comparable to that of the rabbits. Thus, it is highly recommended to perform more experiments on bigger size animals, such as primates, to generalize the results to human beings.

Moreover, despite the great number of studies investigating nerve conduits for peripheral nerve regeneration, many problems still remain in this field and researchers cannot reach a consensus to resolve these issues.

\section{Conclusion}

Some grade of functional recovery was observed with partial repair in a rabbit model, however, further studies need to evaluate the exact outcome.

\section{Acknowledgment}

This project was supported by a grant obtained from Iran University of Medical Sciences, Tehran, Iran. The authors extend their gratitude to Mina Khosravi (Medical Student at Iran University of Medical Sciences), Faezeh Javadi (Medical Student at Iran University of Medical Sciences), and Freshteh Khaleghi (Medical Student at Iran University of Medical Sciences).

\section{Conflict of Interests}

The authors declare that they have no competing interests.

\section{References}

1. Giovanoli P, Koller R, Meuli-Simmen C, RabM, Haslik W, Mittlböck $\mathrm{M}$, et al. Functional and morphometric evaluation of end-toside neurorrhaphy for muscle reinnervation. Plast Reconstr Surg. 2000; 106: 383-92.

2. Viterbo F, Trindade JC, Hoshino K, Mazzoni Neto A. End-to-side neurorrhaphy with removal of the epineurial sheath: an experimental study in rats. Plast Reconstr Surg. 1994; 94: 1038-47.

3. Kanje M, Arai T, Lundborg G. Collateral sprouting from sensory and motor axons into an end to side attached nerve segment. Neuroreport. 2000;3(11): 2455-9.

4. Yamauchi T, Maeda M, Tamai S, Tamai M, Yajima H, Takakura Y, et al. Collateral sprouting mechanism after end-to-side nerve repair in the rat. Med Electron Microsc. 2000;33:151-6.

5. Hayashi A, Yanai A, Komuro Y, Nishida M, Inoue M, Seki $T$. Collateral sprouting occurs following end-to-side neurorrhaphy. Plast Reconstr Surg. 2004;114:129-37.

6. Bontioti E, Kanje M, Lundborg G, Dahlin LB. End-to-side nerve repair in the upper extremity of rat. J Peripher Nerv Syst. 2005;10: 58-68.

7. Schmidhammer R, Redl H, Hopf R, van der Nest DG, Millesi H. Synergistic terminal motor end-to-side nerve graft repair: investigation in a nonhumanprimate model. Acta Neurochirurg Suppl. 2007;100(1,3):97-101.

8. Franciosi LF, Modestti C, Mueller SF. Neurotization of the biceps muscle by end-to-side neurorraphy between ulnar and musculocutaneous nerves. A series of five cases. Chir Main. 1998; 17:362-7.

9. Pondaag W, Gilbert A. Results of end-to-side nerve coaptation in severe obstetric brachial plexus lesions. Neurosurgery. 2008 Mar;62: 656-63.

10. Bain JR, Mackinnon SE, Hunter DA. Functional evaluation of complete sciatic, peroneal, and posterior tibial nerve lesions in the rat. Plast Reconstr Surg. 1989;83:129-38.

11. Nectow AR, Marra KG, Kaplan DL. Biomaterials for the development of peripheral nerve guidance conduits. Tissue Eng Part B Rev. 2012;18:40-50.

12. Clavijo-Alvarez JA, Nguyen VT, Santiago LY, Santigo LY,Doctor JS, Lee WP, Mara KJ. Comparison of biodegradable conduits within aged rat sciatic nerve defects. Plast Reconstr Surg. 2007;119:18391851.

13. Shin RH, Friedrich PF, Crum BA, Bishop AT, Shin AY. Treatment of a segmental nerve defect in the rat with use of bioabsorbable synthetic nerve conduits: a comparison of commercially available conduits. J Bone Joint Surg Am. 2009;91:2194-2204. 